\title{
Colour Information Loss due to Decoherence in Vacuum
}

\author{
Viatcheslav Kuvshinov ${ }^{1, \star}$ and Eugene Bagashov ${ }^{1}$ \\ ${ }^{1}$ Joint Institute for Power and Nuclear Research - Sosny of National Academy of Sciences of Belarus, Minsk, \\ P/O box 119
}

\begin{abstract}
The model of stochastic vacuum of quantum chromodynamics might be used for an alternative treatment of subhadronic processes. The possibility of considering the stochastic vacuum as an environment for colour charges is discussed. It is shown that the interaction of colour particles with the stochastic vacuum leads to the decoherence process and the loss of information on the initial quantum state of the particle, which gives a new perspective regarding the confinement of quarks phenomenon.

The mentioned effect is demonstrated in cases of a single particle and a superposition of colour charges. Generalization on the multiparticle case is proposed.

Quantum characteristics (purity and von Neumann entropy) are used to analyse the process of interaction. On the base of von Neumann entropy the measure of quantum information is proposed.
\end{abstract}

\section{Introduction}

Interactions of some quantum system with the environment can be effectively represented by additional stochastic terms in the Hamiltonian of the system. The density matrix of the system is then obtained by averaging with respect to degrees of freedom of the environment. Interactions with the environment result in decoherence and loss of quantum superpositions. Information on the initial state of the quantum system is lost after sufficiently large time.

Quantum decoherence is the loss of coherence or ordering of the phase angles between the components of a system in a quantum superposition. Decoherence occurs when a system interacts with its environment in a thermodynamically irreversible way. This process can be viewed as the loss of information on a system. Dissipation is a decohering process by which quantum states are changed due to entanglement with an external thermal bath.

\section{Stochastic QCD Vacuum}

The model of stochastic vacuum of quantum chromodynamics (QCD) is one of the popular phenomenological models which explains quark confinement (Wilson loop decreasing), string tensions and field congurations around static colour charges.

In this model only the second field correlators are important and the others are negligible [1] (Gauss domination). It has been confirmed by lattice calculation [2]. The most important evidence for this is Casimir scaling.

\footnotetext{
^e-mail: kuvshinov2003@gmail.com
} 
The stochastic vacuum approach is based on the assumption that one can calculate vacuum expectation values of gauge invariant quantities as expectation values with respect to some well-behaved stochastic gauge field. It is known that such vacuum provides confining properties, giving rise to QCD strings with constant tension at large distances $[1,3,4]$.

\subsection{QCD Vacuum as Environment for Colour Particles}

We consider QCD stochastic vacuum as the environment for colour quantum particles. To get the density matrices of those particles we average over the external QCD stochastic vacuum implementations.

Thus, instead of considering nonperturbative dynamics of Yang-Mills fields one introduces external environment (in our case represented by QCD stochastic vacuum) and average over its implementations. As a consequence, we obtain decoherence, loss of quantum superpositions and information, and confinement of colour states.

White objects can be obtained as colourless mixtures of states described by the diagonal density matrix as a result of evolution in the QCD stochastic vacuum.

\section{Decoherence of Colour States}

Consider propagation of heavy spinless colour particle along some fixed path $\gamma$. The amplitude of such process is obtained by parallel transport [5-7]

$$
\begin{gathered}
\partial_{\mu}|\phi\rangle=i \hat{A}_{\mu}|\phi\rangle, \\
|\phi(\gamma)\rangle=\mathcal{P} \exp \left(i \int_{\gamma} d x^{\mu} \hat{A}_{\mu}\right)\left|\phi_{i n}\right\rangle,
\end{gathered}
$$

where $\mathcal{P}$ is the path ordering operator and $\hat{A}_{\mu}$ is the gauge field vector. In order to consider mixed states we introduce the colour density matrix, taking into account both colour particle and QCD stochastic vacuum (environment):

$$
\hat{\rho}(\text { loop }, \gamma \bar{\gamma})=<|\phi(\gamma)\rangle\langle\phi(\gamma)|>,
$$

Here we average over all implementations of stochastic gauge field (environment degrees of freedom) - and decoherence arises due to interaction with environment.

In the model of QCD stochastic vacuum only expectation values of path ordered exponents over closed paths are defined (in order to keep the gauge invariance). Closed path corresponds to a process in which the particle-antiparticle pair is created, propagates and finally annihilates.

With the help of (1), (2), (3) we can obtain the expression for density matrix [5, 7]:

$$
\hat{\rho}(\text { loop }, \gamma \bar{\gamma})=N_{c}^{-1}+\left(\left|\phi_{\text {in }}\right\rangle\left\langle\phi_{\text {in }}\right|-N_{c}^{-1}\right) W_{a d j}(\gamma \bar{\gamma}) .
$$

Here $N_{c}$ is the number of colours.

Colour density matrix in colour neutral stochastic vacuum can be decomposed into the pieces that transform under trivial and adjoint representations $[5,7]$

$$
\hat{\rho}_{1}=N_{c}^{-1} \hat{I}+\rho_{1}^{a} \hat{T}_{a},
$$

and Wilson loop in fundamental representation is [1]

$$
W_{\text {fund }}(\text { loop, } \gamma \bar{\gamma})=\left\langle\operatorname{Tr} \mathcal{P} \exp \left(\int_{\text {loop }, \gamma \bar{\gamma}} i \hat{A}_{\mu} d x^{\mu}\right)\right\rangle \text {. }
$$


In confinement region Wilson loop decays exponentially with the area spanned by loop, so for the rectangular loop spanning the time interval $T$ and distance $R$ we get:

$$
\begin{gathered}
\hat{\rho}(\text { loop }, \gamma \bar{\gamma})=\hat{I} N_{c}^{-1}+\left(\hat{\rho}_{i n}-\hat{I} N_{c}^{-1}\right) \exp \left(-\sigma_{a d j} R T\right), \\
\hat{\rho}(\gamma: R T \rightarrow \infty)=N_{c}^{-1} \hat{I},
\end{gathered}
$$

where $\hat{I}$ is the unit operator, $\sigma_{a d j}=\sigma_{\text {fund }} G_{a d j} G_{\text {fund }}^{-1}$ is the string tension in the adjoint representation, $G_{a d j}, G_{f u n d}$ are the eigenvalues of quadratic Casimir operators. Under Gaussian dominance string tension is

$$
\sigma_{\text {fund }}=\frac{g^{2}}{2} l_{\text {corr }}^{2} F^{2}
$$

$g$ is coupling constant, $l_{\text {corr }}$ correlation length in the QCD stochastic vacuum, $F$ - average of the second cumulant of curvature tensor $[2,4]$.

As follows from (8) all colour states are mixed with equal probabilities and all information on initial color state is lost.

\subsection{Decoherence Characteristics}

The decoherence rate of transition from pure colour states to white mixture state can be estimated on the base of purity [8]:

$$
P=\operatorname{Tr} \hat{\rho}^{2}
$$

This characteristic represents the closeness of a quantum state to a pure one. In our case,

$$
P=N_{c}^{-1}+\left(1-N_{c}^{-1}\right) \exp \left(-2 \sigma_{\text {fund }} G_{a d j} G_{\text {fund }}^{-1} R T\right) .
$$

When $R T$ tends to $0, P \rightarrow 1$, which corresponds to a pure state. When the composition $R T$ tends to infinity, the purity tends to $N_{c}^{-1}$, which corresponds to the white mixture [7].

The rate of purity decrease is

$$
T_{d e c}^{-1}=-2 \sigma_{\text {fund }} G_{a d j} G_{f u n d}^{-1} R .
$$

Left side of the equation is the inverse characteristic time of decoherence proportional to QCD string tension and distance $R$. It can be inferred from (4) and (11) that the larger is the distance between particle and antiparticle, the quicker the initial state tends to white mixture as a result of interaction with the QCD stochastic vacuum. Thus white states can be obtained as a result of decoherence process. The information of quark colour states is lost in hadrons due to interactions between quarks and confining non-Abelian gauge fields.

Von Neumann entropy can be used as a measure of the loss of information:

$$
S=-\operatorname{Tr}(\hat{\rho} \ln \hat{\rho}) .
$$

Initial density matrix gives us $S=0$ and we will have $S=\ln N_{c}$ for large $R T$.

In order to obtain a more suitable characteristic to work with, we might want to define the information on the basis of the given entropy parameter. As it can be seen, the entropy ranges from 0 (for a pure, unperturbed quantum state, which in our approach corresponds to the maximum amount of quantum information) to $\ln N_{c}$. So it would be quite natural to define the information measure as

$$
I=\frac{\ln N_{c}-S}{\ln N_{c}}=1-\frac{S}{\ln N_{c}} .
$$


So the overall range of this measure is $[0,1]$ : in case of zero entropy it is equal to 1 and in case of maximum entropy it is equal to 0 . The latter case corresponds to the asymptotically big values of $R T$ (Wilson loop area).

Thus, as we might see, during the interaction with QCD vacuum the entropy increases and the information is being lost.

\subsection{Interaction of Colour Superposition with QCD Vacuum}

When the initial (pure) colour state is a superposition of colour states, the process of the interaction with vacuum can be expressed with the following equations [9]:

$$
\begin{gathered}
\left|\phi_{\text {in }}\right\rangle=\alpha|1\rangle+\beta|2\rangle+\gamma|3\rangle, \\
|\alpha|^{2}+|\beta|^{2}+|\gamma|^{2}=1 .
\end{gathered}
$$

The corresponding density matrix is

$$
\begin{gathered}
\hat{\rho}_{\text {in }}=\left|\phi_{\text {in }}\right\rangle\left\langle\phi_{\text {in }}\right|, \\
\hat{\rho}_{\text {in }}=\left(\begin{array}{lll}
|\alpha|^{2} & \alpha \beta^{*} & \alpha \gamma^{*} \\
\alpha^{*} \beta & |\beta|^{2} & \beta \gamma^{*} \\
\alpha^{*} \gamma & \beta^{*} \gamma & |\gamma|^{2}
\end{array}\right) .
\end{gathered}
$$

After the integration and averaging we arrive at the same result as in (7) and thus in the confinement region $(R T \rightarrow \infty)$ the same diagonalization of the density matrix would be observed (see 8 ). Thus density matrix during the interaction with QCD vacuum would transform in the following way:

$$
\left(\begin{array}{ccc}
|\alpha|^{2} & \alpha \beta^{*} & \alpha \gamma^{*} \\
\alpha^{*} \beta & |\beta|^{2} & \beta \gamma^{*} \\
\alpha^{*} \gamma & \beta^{*} \gamma & |\gamma|^{2}
\end{array}\right) \rightarrow\left(\begin{array}{ccc}
N_{c}^{-1} & 0 & 0 \\
0 & N_{c}^{-1} & 0 \\
0 & 0 & N_{c}^{-1}
\end{array}\right) .
$$

(In this particular case $N_{c}=3$ ). Purity changes from 1 (as the density matrix $\rho_{\text {in }}$ is idempotent) to $N_{c}^{-1}$, signaling the departure of our system from the pure state and turning into fully mixed state. And the information measure introduced in Sec. 3.1 changes from 1 to 0, which corresponds to the information loss.

Thus the interaction of an arbitrary colour superposition with the QCD stochastic vacuum at large distances leads to an emergence of a mixed state with equal probabilities for different colours (without non-diagonal terms in the corresponding density matrix).

\section{Multiparticle States and QCD Vacuum}

Density matrix of a system is dependent on the quantity of its degrees of freedom, which is dependent on the number of particles in the system. Accordingly, the density matrix dimesions for multiparticle states would be increased from $\left(N_{c}\right) \mathrm{x}\left(N_{c}\right)$ to $\left(N_{c}^{N_{p}}\right) \mathrm{x}\left(N_{c}^{N_{p}}\right)$, where $N_{p}$ is the number of particles. It is necessary, therefore, to make the corresponding changes in all the formulas that were considered for a single-particle colour superposition. So the expression (7) in the multiparticle case would look like

$$
\hat{\rho}=N_{c}^{-N_{p}} \hat{I}+\left(\hat{\rho}_{i n}-N_{c}^{-N_{p}} \hat{I}\right) \exp \left(-\sigma_{a d j} R T\right) .
$$

And the expression (8) can be written as

$$
\hat{\rho}(\gamma: R T \rightarrow \infty)=N_{c}^{-N_{p}} \hat{I} .
$$


Table 1. Purity and entropy of different states of multiparticle system before the interaction with vacuum

\begin{tabular}{lllll}
\hline State: & pure separable & mixed separable & pure entangled & mixed entangled \\
\hline $\mathrm{P}$ (purity) & 1 & $\frac{1}{N_{c}^{N_{p}}} \leq P<1$ & 1 & $\frac{1}{N_{c}^{N_{p}}}<P<1$ \\
S (entropy) & 0 & $0<S \leq N_{p} \ln N_{c}$ & 0 & $0<S<N_{p} \ln N_{c}$ \\
\hline
\end{tabular}

Table 2. Purity and entropy of different states of multiparticle system after the interaction with vacuum

\begin{tabular}{lllll}
\hline State: & pure separable & mixed separable & pure entangled & mixed entangled \\
\hline $\mathrm{P}$ (purity) & $\frac{1}{N_{c}^{N_{p}}}$ & $\frac{1}{N_{c}^{N_{p}}}$ & $\frac{1}{N_{c}^{N_{p}}}$ & $\frac{1}{N_{c}^{N_{p}}}$ \\
$\mathrm{~S}$ (entropy) & $N_{p} \ln N_{c}$ & $N_{p} \ln N_{c}$ & $N_{p} \ln N_{c}$ & $N_{p} \ln N_{c}$ \\
\hline
\end{tabular}

The purity (10) would change to:

$$
\mathrm{P}=N_{c}^{-N_{p}}+\left(1-N_{c}^{-N_{p}}\right) W_{a d j}^{2}(\gamma),
$$

with maximum von Neumann entropy (13) being:

$$
S=N_{p} \ln N_{c} .
$$

The proposed information measure (14) might then be generalized as

$$
I=1-\frac{S}{N_{p} \ln N_{c}}
$$

Here is the list of possible types of states, in which the system may be found (excluding some specific types e.g. as the squeezed states):

- By purity: 1) pure; 2) mixed.

- By separability: 1) separable; 2) non-separable (entangled).

Table 1 represents the quantum characteristics for the four types of states (before the interaction with vacuum). The strictness of the inequalities in the last column is due to the fact that a fully mixed state is always separable.

The same quantities after the interaction are shown in table 2 .

Thus we can see that the final state is defined by a number of particles (and the number of possible colours for any individual particle) in the system. But in any case the entropy during the interaction with QCD vacuum rises to its maximum value and the information is being lost in this process. In the limit $W(\gamma) \rightarrow 0$ we get fully mixed state of a form (21).

\section{Conclusions}

Vacuum of quantum chromodynamics can be considered as environment (in the sense of quantum optics) for colour particles. Density matrix, purity and von Neumann entropy for colour particles depend on Wilson loop averaged through QCD vacuum degrees of freedom.

In the case of of stochastic (not coherent) QCD vacuum (only correlators of the second order are important) in confinement region (Wilson loop decays exponentially) we have decoherence of pure colour states into a mixed white states, while purity decays exponentially (decay rate $=$ string tension).

For multiparticles (pure separable, mixed separable and nonsepaparable (entangled) states) when $R T \rightarrow \infty$ we obtain diagonalization of density matrix, decreasing of purity and increasing of von Neumann entropy. 


\section{References}

[1] Y. A. Simonov. The Confinement. Uspekhi Fizicheskih Nauk. 4 (1996) (in Russian).

[2] A. D. Giacomo, H. Dosch, V. I. Shevchenko, and Y. A. Simonov, Physics Reports 372, no. 4, 319-368 (2002).

[3] J. Ambjørn, P. Olesen. On the Formation of a Color Magnetic Quantum Liquid in QCD. Nuclear Physics B. 170, no. 1, 60-78 (1980).

[4] D. S. Kuzmenko, Y. A. Simonov, et al. Vacuum, Confinement and QCD Strings in the Vacuum Correlator Method. Uspekhi Fizicheskih Nauk. 174, no. 1 (2004) (in Russian).

[5] V. Kuvshinov, A. Kuzmin. Gauge Fields and Theory of Deterministic Chaos. (Belorussian Science, Minsk, 2006, in Russian).

[6] V. Kuvshinov, P. Buividovich. White Mixed States in QCD Stochastic Vacuum. Nonlinear Phenomena in Complex Systems. 8, no.3, 313-316 (2005).

[7] V. Kuvshinov, P. Buividovich. Decoherence of Quark Colour States in QCD Vacuum. Acta Physica Polonica B. (Proceedings Supplement), 1, no. 3, 579-582 (2008).

[8] F. Haake. Quantum Signatures of Chaos. (Springer-Verlag, Berlin, 1991).

[9] V. I. Kuvshinov, E. G. Bagashov. Evolution of colour superposition in the stochastic QCD vacuum. Nonlinear Phenom. Complex Syst., 16, No. 3, 242-246 (2013). 